\title{
Development of a standardized method to evaluate the protective efficiency of cosmetic packaging against microbial contamination
}

Chloe Catovic

University of Rouen

Sylvia Martin

Shiseido Co Ltd

Sephane Desaint

Yves Rocher Innovation \& Developpement

Christine Borges

RPC Promens

Helene Lesouhaitier

Johson \& Johson Santé Beauté France

Florence Roullet

Aptar Beauty \& Home

Nadine Bresciani

Chanel Parfum Beauté

Anne-Marie Jouault

Sisley

Valerie Poulet

Clarins

Joelle Luc

Pierre Fabre Dermo-Cosmetique

Valérie Joulia

Pierre Fabre Dermo-Cosmetique

Alain Jupin

Albea

Christophe Masson

Cosmetic Valley

Alain Crozier

Clean Cosmetic Consulting

Marc G.J. Feuilloley ( $\nabla$ marc.feuilloley@univ-rouen.fr )

Universite de Rouen https://orcid.org/0000-0001-6467-4336 


\section{Original article}

Keywords: cosmetic packaging, microbial contamination, quality, evaluation

Posted Date: April 28th, 2020

DOl: https://doi.org/10.21203/rs.3.rs-19549/v3

License: (c) (i) This work is licensed under a Creative Commons Attribution 4.0 International License. Read Full License

Version of Record: A version of this preprint was published at AMB Express on April 24th, 2020. See the published version at https://doi.org/10.1186/s13568-020-01016-4. 


\section{Abstract}

Doubts surrounding the potential adverse effects of antimicrobial preservatives have modified the demand of consumers, who increasingly insist on the production of low-level and even preservative-free cosmetics. Protection of the product against microbial contamination is therefore focused on the packaging. This has prompted the emergence of a highly diverse array of so-called "protective", "overprotective", and "barrier" packaging. However, these designations are not normalized and the choice of the right packaging adapted to each cosmetic product is still essentially empirical, hazardous, and time consuming. The Cosmetic Valleys cluster has launched a commission to define a complete and experimentally-validated method to classify the level of protection of cosmetic packaging against microbial contamination. As reported herein, this required the development a specific bacteriostatic medium that can be used for seven days and an in vitro procedure that reproduces in-use contamination and consumer practices. Based on tests performed on over 800 packages of different origin and performance characteristics, we propose a classification, divided into six grades, to differentiate the protective efficiency of cosmetic packaging. This work can be considered as a first step towards a regulatory text.

\section{Introduction}

Demonstration of the health and environmental risks associated with the presence of preservatives in personal-care products and cosmetics has prompted the emergence of low-level and even preservativefree products (Halla et al., 2018). As such formulations are particularly sensitive to microbial contamination, protection of the product has focused on the packaging. The role of packaging in the preservation of cosmetics is well known and the packaging itself is considered to be an essential part of the final product in regulatory texts (Lundov et al.,2009; Regulation EC 1223/2009). Consequently, a large range of high-technology primary packaging (airless, air filter, shutter equipped devices, etc.) to provide both physical and microbial protection of the product has been developed (Crozier, 2018a). Such packaging has essentially two functions, protecting the product in the device by limiting all potential retro-contamination and ensuring delivery of minimally-contaminated and safe doses throughout the shelf life of the product. These objectives are somewhat contradictory, as it is particularly difficult to associate increased hermetic closure and regular delivery of the content throughout the life of the product, except for single use devices, which are faced with other problems, such as increased containcontainer interactions (Feuilloley \& Orange, 2018) and ecological compatibility (Thompson et al., 2009). In addition, delivery of a dose implies the potential accumulation of remaining product inside (dead space zones) and/or even outside of the device, with a high risk of contamination when the product is not selfprotected.

As the packaging is an intrinsic element of low-level and preservative-free cosmetic products, it is essential to adapt its performance to the protection of the formulation to insure sanitary safety. Conversely, as certain active cosmetic ingredients also have antimicrobial activity, even in the absence of added preservatives (Papageorgiou, 2010; Herman et al., 2013), the formula can provide a certain 
amount of protection against microbial contamination and the pack should be adapted to this situation to avoid over-quality costs. Protocols have been proposed to evaluate the microbial protection provided by cosmetic packaging (Devlieghere et al., 2015; Briasco et al., 2016). However, the packaging and formulation are generally produced by different partners and, in the absence of regulatory texts and even technical tools that make it possible to define a clear hierarchy from low to overprotective and barrier packaging, selecting the correct association is essentially empirical and potentially hazardous.

The Cosmetic Valley cluster, the world's leading center for resources in perfumery and cosmetics, offers the possibility to associate professionals of the packaging and cosmetic industries with academic investigators to define, for the first time, a technically functional harmonized procedure that could be applicable to all industrial partners and potentially translated into a future harmonized regulatory standard. Here, we describe the logical and technical approach used to define such a procedure, the experimental studies conducted to overcome fundamental technical obstacles, and the tests performed for its validation.

\section{Materials And Methods}

\section{Definition of the target and regulatory context}

A "Guideline for the evaluation of physical antimicrobial protection provided by packaging" was developed for the comparison of the performance of commercially available cosmetic packaging, taking into account the physical properties (fluidity) of the potential final formulation and, independently, its composition. This procedure can also be used to compare devices under development, although in this case, the true level of protection would remain relative. The results should be integrated into a microbial risk assessment procedure, as described by ISO 11930 (2019) and ISO 29621 (2017). It is essential that it be possible for the tests to be performed by the packaging and cosmetic industries. Thus, as the staff is normally trained to work under sterile conditions and has access to the necessary equipment, the biological risk of all microbial strains had to be level two or lower (European Community classification, 2005). One of the goals was to also take into account the true practices of the consumer, as they are the principal source of contamination. Thus, a specific in vitro contamination procedure was developed to mimic normal use.

\section{Selected bacterial model}

To be economically realistic, it became rapidly apparent that the procedure should provide results using a single model microorganism. In addition to being level two or below, the following key criteria were retained for its selection:

- Presence in the microbial library of most industrial sites

- Aerobic and easy to grow

- Average size to avoid potential under estimation of the protective performance of the packaging 
- Frequently found in the environment and potentially on human skin

- Mobile, so that it can diffuse throughout dead spaces and be detectable

Considering all these elements, the species Pseudomonas aeruginosa was selected. All tests were realized using the strain ATCC® CRM-9027. Equivalent strains $\mathrm{CIP} \circledast 82.118, \mathrm{NCIMB} \circledast 8626$, NBRC® 13275 , or KCTC® 2513 should also be usable.

\section{Type of packaging and sampling procedure}

Predictable low-level non-protective packaging against microbial contamination, such as pots or open tubes, which provide no protection to the contents, were excluded from the study. At the other end of the protection spectrum, hermetic, sterile, single-use packaging, corresponding to pharmaceutical-quality products, was also considered to be out of the scope of the present study.

It was thus decided to develop the present procedure for two types of devices:

- Overprotective packaging

- Absolute barrier packaging

Given their production mode, it was decided that the tests had to be conducted on a minimum of 50 randomly selected devices to reach reliable statistical values. Before entering into the testing procedure all devices were decontaminated using a technique adapted to their composition (ionization, autoclaving, etc.). All devices were filled under sterile conditions and control sterility tests were performed.

\section{Testing media}

Overprotective packaging is not meant to be totally hermetic to contamination. As one of the targets of the protocol was to take into consideration the real "in use" practices of the consumer, it was decided that the contamination procedure should be repeated for at least a week. The contamination level of distributed doses can be measured immediately, but that of the formulation present in the reservoir of the device can only be measured after opening. Given that bacteria can multiply very rapidly, it would be impossible to determine at which time of the test the initial contamination occurred. Thus, it was necessary to develop a bacteriostatic medium for $P$. aeruginosa. In addition, this bacteriostatic medium had to be supplemented with a reticulation agent to adapt the viscosity, as the performance of the packaging is adapted to the mean fluidity of cosmetic creams.

Barrier packaging should normally block all bacterial contamination, while delivering repeated doses. In this respect, perfectly safe absolute barrier packaging is yet to be developed, but very high efficiency barrier devices, adapted to low protection formulae, have been marketed. Therefore, we tested such packaging using a classical fertile medium for $P$. aeruginosa. However, such packaging is also designed to be used with cosmetic creams. Thus the classical fertile media had to be formulated using a reticulation agent, as it is normally too fluid. 


\section{Contamination procedure}

To reproduce normal use of the device, the packaging was exposed to contamination by wiping the nozzle while dispensing a dose on a sterile compress (30 g/m², NF-EN 29073-1) impregnated with bacteria at a concentration of $10^{6} \mathrm{CFU} / \mathrm{cm}^{2}$ (Figure 1). This bacterial concentration was selected to be equivalent to the mean bacterial load of human skin (Wilson, 2005). The compress was open to form a $100 \times 200-\mathrm{mm}$ rectangle and inserted into a sterile stomacher bag to ensure homogeneous contamination. The bacterial solution $\left(5 \mathrm{~mL}, 10^{8} \mathrm{CFU} / \mathrm{mL}\right)$ was distributed over the entire surface of the pad and after closure of the bag, the liquid was spread by exerting a gentle pressure from the center to the periphery. This procedure was validated by testing colored media supplemented with phenol red to verify the homogeneous distribution of the solution.

The contamination simulation was conducted over eight days at room temperature, as presented in Table 1. Given that days 1 and 2 were used for carrying out the controls, filling the packaging, and sub-culturing the bacteria, the true simulation procedure started on day 3 by controlling the sterility of the package and exposition to the contaminated pad in the morning and afternoon at 6-h intervals (minimum). Thereafter, the packaging was exposed twice a day at $6 \mathrm{~h}$ intervals to the contamination from days 4 to 7 , as shown in Figure 1, and two doses were collected. A first dose was also collected on day 3 before the first daily contamination and was used as a control. On day 8 , the bacteria in $1 \mathrm{~g}$ of delivered medium were enumerated. Then, the packaging was opened under sterile conditions and an aliquot of the formulation remaining in the container was collected and plated on Petri dishes to assess potential contamination that developed in the container.

For determination of the contamination level of the delivered doses, each dose was weighed and diluted 1/10 and 1/100 (w/v) in Eugon LT100 medium (Fisher Scientific) before plating on tryptase soya broth (TSA)-agar solid medium. Eugon LT100 was necessary to neutralize the potential remaining bacteriostatic activity of the medium used to test the overprotective packaging. CFU were counted after 48 to $72 \mathrm{~h}$ of incubation at $32.5 \pm 2.5^{\circ} \mathrm{C}$.

\section{Results}

\section{Formulation of the bacteriostatic and fertile media}

There are many media available for increasing the cultivability of bacteria. Conversely, bacteriostatic media are rarely available, particularly for a versatile species such as $P$. aeruginosa. Moreover, although certain media have bacteriostatic activity lasting for 48 or even $72 \mathrm{~h}$ (Zwisler laboratorium $₫$ medium), we required a medium that remained bacteriostatic over a minimum of seven days to avoid any artefacts in the evaluation of the packaging. Another challenge was the obligation to adapt the fluidity of the medium to be compatible with the mean fluidity of the cosmetic formulations classically employed in the packaging. All compounds also had to be of limited cost and compatible with the safety rules of industrial companies. 
Starting from the composition of the Zwisler laboratorium ${ }^{\circledR}$ medium, we performed a series of tests to develop a medium with bacteriostatic activity on $P$. aeruginosa that lasted for more than one week using media with the basic formula:

- DPBS (Dulbecco's Phosphate-Buffered Saline) (Thermo Fisher) $900 \mathrm{~mL}$

- Glycerol (Carl Roth) $100 \mathrm{~mL}$

- MgSO4 (anhydrous) (Sigma Aldrich) $4 \mathrm{~g}$

- Phenol red (Merck Millipore) $5 \mathrm{mg}$

- Low viscosity carboxymethyl cellulose (Sigma Aldrich ) $0 \mathrm{~g}$

- Bacteriostatic agent (varying percentage for tested molecules)

All tested media were inoculated at day 0 with $100 \mathrm{UFC} / \mathrm{g}$ of $P$. aeruginosa. For each bacteriostatic agent tested, the evolution of the contamination was measured over 7 days and was expressed as the logarithmic variation of the initial inoculum ( $\Delta$ log colony-forming unit). The principal bacteriostatic agents studied are presented in Figures $\mathbf{2}$ (acids) and in Figure $\mathbf{3}$ (common organic preservatives). As previously mentioned, these substances were selected as they are low cost and present in most industrial companies. Hydrochloric acid (Figure 2A), citric acid (Figure 2B), and sorbic acid (Figure 2C) were tested based on the hypothesis that a decrease in $\mathrm{pH}$ affects the growth of $P$. aeruginosa (Sporer al., 2017). Boric acid (Figure 2D) was tested as, in addition to its acidifying effect, borate ions can also affect bacterial growth (Lum \& Meers, 1989). Other common preservatives were also tested, such as phenoxyethanol (Figure 3A) used in cosmetics for its broad preservative activity (ANSM, 2012). Sodium benzoate (Figure 3B) a food preservative also used in pharmaceutical formulations was included in the tests.. Methyl isothiazolinone (MIT) (Figure 3C), a powerful synthetic biocide, was also tested, despite its known skin sensitization activity. We also decided to test methyl paraben (methyl parahydroxybenzoate MPOB), the E218 food preservative (Figure 3D). These compounds were studied over a wide range of doses, although only the more relevant results are shown in the figures. Caprilyl glycol, a skin conditioning agent with antimicrobial activity, and nalidixic acid, a DNAgyrase inhibitor known to block bacterial division were also tested in preliminary studies (data not shown).

Hydrochloric acid ( $\mathrm{pH}=5.39$ ) (Figure 2A) showed inhibitory activity, but this effect was lost with very limited increases in the $\mathrm{pH}$. At $\mathrm{pH}=5.45$ and above, the contamination level of the medium at day 1 was too high to be determined $(\infty)$. Citric acid (Figure 2B) partially blocked the growth of $P$. aeruginosa at pHs $=4.79$ and 4.81 but acted as an inhibitor at $\mathrm{pH}=4.94$. Sorbic acid (Figure $2 \mathrm{C}$ ) was completely ineffective in blocking the growth of $P$. aeruginosa at any concentration tested (0.05 to $0.1 \%$ ). Boric acid (Figure 2D) showed good bacteriostatic activity on $P$. aeruginosa, particularly when used at $0.26 \%(\mathrm{pH}=6.00)$ or $0.84 \%(\mathrm{pH}=5.41)$. At higher concentrations boric acid was lethal to the bacteria. The response of $P$. aeruginosa to phenoxyethanol (Figure 3A) was unexpected. Except at the highest concentrations used ( $0.35 \%$ and above), the bacteria grew for one or two days but then stopped, probably due to the toxic effect of this molecule. Up to a concentration of $0.045 \%$, sodium benzoate (Figure $3 B$ ) was only able to delay the onset of $P$. aeruginosa multiplication, and no detectable viable bacteria were detected at day 7 
at higher dose (0.05\%). Methyl paraben (POBM) (Figure 3C) also showed a varying response, ranging from a limited inhibition of bacterial growth at $0.1 \%$ to a complete inhibition at $0.15 \%$. At day 7 , the effect of methyl isothizolinone (MIT) (Figure 3D) passed from the total absence of inhibition at $0.001 \%$ to total inhibition at $0.002 \%$. Thus, except for boric acid, all tested compounds were unable to inhibit the development of $P$. aeruginosa over 7 days or the range of their effective concentration was so narrow (particularly $\mathrm{pH}$ values) that their use in reproducible tests was impractical. Finally, although the use of boric acid is restricted because its classification by the EU as a carcinogen, mutagen, and reprotoxic (CMR) 1B or 2, depending on its concentration, it was selected for production of the bacteriostatic medium. However, the viscosity of the first bacteriostatic medium was too low, although it included carbomethylcellulose. Thus, a second series of tests was carried out using various reticulation agents. The viscosity of the formula was estimated visually (Table 2) and that of the more suitable solutions measured using a Viscotester IQ Haake rheoviscosimeter with coaxial cylinders and a CC25 DIN/Ti gap. Considering the range of the mean fluidity $\left(18,000 \pm 3,000 \mathrm{mPas}\right.$ at $25^{\circ} \mathrm{C}$, shear $\left.15^{\mathrm{s}-1}\right)$ of most typical cosmetic lotions, hydroethylcellulose (HEC, $25 \mathrm{~g} / \mathrm{L}$ ) was selected to formulate the bacteriostatic medium. The activity of this medium was verified using a $100 \mathrm{CFU} / \mathrm{mL} P$. aeruginosa inoculum, as any modification of the formula can influence the bacteriostatic properties. It was validated based on a maximal variation $<50 \%$ (0.3 log), according to standard guidelines (ISO 21149).

As previously mentioned, selecting a fertile medium was a simpler task. It included $\mathrm{MgSO}_{4}$, glycerol, and phenol red at the same concentrations as those used for the bacteriostatic medium to keep the two formulations similar. DPBS was replaced by Trypcase Soja Broth (TSB) to favor bacterial growth. However, the use of TSB modified the fluidity of the medium and it was necessary to increase the amount of reticulation agent (HCE) to $25.7 \mathrm{~g} / \mathrm{L}$ to preserve the same rheological properties.

\section{Comparative tests of overprotective and barrier packaging}

A total of 16 series of 50 packages were submitted for in-use tests in duplicate by five different cosmetics producers. These tests were performed by seven different operators. They included five types of packaging of different protective levels (presumably overprotective or barrier) from three different producers. The packagess were anonymized and identified by letters. They were transmitted without the origin of reference to the laboratory in charge of the tests. The results are presented in Table 3. One of the packages (A) provided a limited level of protection, as all delivered doses 1 and 2 were contaminated, generally with a high number of microorganisms (less than $30 \%$ of the delivered doses contained $<1,000$ UFC of contamination). Package B provided better protection, although some of the delivered doses contained $>1,000$ UFC of contamination. Package $D$ gave better results, in which all delivered doses for one series of tests showed $<1,000$ UFC of contamination, but the performance was irregular. Packages $C$ and $\mathrm{E}$ showed the best level of protection in these tests. As expected by overprotective or barrier packs, all the tested devices provided complete protection of the reservoir content.

\section{Discussion}


Faced with the diversity of technical solutions proposed to preserve low-level and preservative-free cosmetic products, both the packaging and cosmetic industries should gain from the existence of harmonized standards. In addition to regulatory texts, several rare studies have been conducted to validate the microbial safety of cosmetic packaging (Crozier, 2018a). However, this study is the first to validate an experimental protocol over a wide range of devices and associated them a classification grid allowing their differentiation based on the degree of microbiological safety conferred by the package.

Pseudomonas aeruginosa was selected as a model because of its listing as a challenge test microorganism (ISO11930 2019) and therefore its presence in the microbial library of most industrial sites. In addition, this bacterium grows easily, it is of average size, is widely distributed throughout the environment, and is mobile due to its polar flagella. This bacterial model has the drawback of being a safety level 2 microorganism and thus requires specific facilities. This was preferred to a ubiquitous safety level 1 species, such as Pseudomonas fluorescens (Bossis et al. 2000), as this bacterium is not generally available and manipulated in industry and our aim was that our approach be applicable for packaging users and producers. The choice of $P$. aeruginosa, aside from and because of its versatility, was also probably at the origin of the difficulty to formulate a medium that preserved its bacteriostatic activity for seven days. This can appear to be trivial, but although bactericidal and fertile medium can be easily found in the literature, this is the first time that an exhaustive study has been performed on a $P$. aeruginosa bacteriostatic medium, particularly one that functions over a long time. The efficiency of boric acid in the formulation of a bacteriostatic medium relative to all other tested substances is supported by its long-standing use as a preservative for the transport of urine samples for clinical observations (Lum and Meers, 1989). Boric acid was more recently proposed for the preservation of urine samples for veterinary applications (Rowlands et al., 2011). The major limitation for the use of boric acid is its classification as hazardous (CMR2 or 1, depending on the exposure concentration) by the European Chemical Agency (ECHA, 2010). Here, boric acid was used at a maximum concentration of $0.485 \%$ (mass/vol). Thus, only manipulation of the bulk material should require CMR protection protocols.

Another important aspect of the protocol concerned the development of an in-use contamination procedure aimed at reproducing the practices of the consumer. This is essential, as contact with the skin and its natural microbiota is the major source of contamination for cosmetic products. In addition, in case of incomplete closure, wiping the outlet can generate limited pressure and favor reflux of the product into the container and contamination of the reservoir. Another problem is that if a significant amount of product remains in the outlet between successive uses and the product is not self-protected, this may be sufficient to permit active bacterial growth and delivery of a contaminated dose. The contamination protocol presented in Figure 1 and Table 1 was established to evaluate the two parameters, i.e. the microbial contamination of the delivered dose and potential contamination of the reservoir. Although it was impossible to investigate the microbiological protection of the packaging throughout its entire lifecycle, including production and storage, an in-use test that spanned seven days appeared to be coherent with validation protocols in regulatory texts. In addition, the validation tests were conducted on a series of 50 packages to account for normal variability of the quality. Indeed, packages are not 
generally produced one by one but using molds with multiple forms and, in spite of controls, not all forms are strictly equivalent in performance.

Given the results, we decided to classify the packaging into six categories ranging from 0 , corresponding to the complete absence of protection, to 5 , providing complete protection. Devices, such as

overprotective and barrier packaging, that were tested in the present study should be classified between 2 and 4, depending on the percentage of contaminated doses measured during the test. In all these devices, the container was absent of contamination. Grade 3 and 4 packaging are differentiated by the tests on fertile medium, which are the most discriminant, and only level 4 can pass the tests with respect to the criteria presented in Figure 4. As this procedure was aimed for products for public use and for use by all interested producers of packaging and cosmetics, it was decided to propose a logotype that should be printed on the identity file of the products. This logotype was deposited to the French organism for the control of intellectual property (INPI) under the property of the Cosmetic Valley cluster. Its use is free but restricted to cosmetic packaging and requires that the device meets the criteria summarized in Table 4.

In conclusion, this study, which has brought together some of the major industrial partners of the cosmetic and packaging industry over the last five years, has resulted in the first complete and experimentally validated protocol that allows the selection of cosmetic packaging as a function of the expected level of microbial protection. This work was presented during a congress to members of the International Organism for Standardization (Crozier 2018; Feuilloley and Roullet, 2018), who will now consider its translation into a new international regulatory text.

\section{Declaration}

Ethics approval and consent to participate. Not applicable, this study did not involve a clinical trial.

Consent for publication. All authors have read and approved the manuscript. LMSM accepts to be responsible for the publication fees.

Availability of data and material. All experimental results are available upon simple demand to the authors. Results on the packaging have been kept anonymized to avoid any commercial application.

Competing interests. The authors have the following interests. Sylvia Martin is employed by Shiseido. Stéphane Desaint is employed by Groupe Rocher. Christine Borges is employed by RPC Promens. Hélène Lesouhaitier is employed by Johnson \& Johnson Santé Beauté. Florence Roullet is employed by Aptar Beauty \& Home. Nadine Bresciani is employed by Chanel Parfum Beauté. Anne-Marie Jouault is employed by Sisley. Joelle Luc is employed by Laboratoires Pierre Fabre Dermo Cosmétique. Christophe Masson is employed by Cosmetic Valley. Alain Crozier is the head of Clean Cosmetic Consulting. There no product or marketing interest to declare. This does not alter the authors' adherence to all policies on sharing data and materials, as detailed online in the guide for authors. Other authors have no conflict of interest to declare. 
Funding. This work was supported by the Evreux Porte de Normandie, Region Normandie, and European Union (FEDER).

Authors' contributions. CC, SM, SD, CB, HL, FL, NB, A-MJ, VP, JL, and VJ contributed to the conception of the approach and conducted experiments. AJ contributed to the analysis of the data. $\mathrm{CM}$ and $\mathrm{AC}$ coordinated the work. AC and MF designed and wrote the manuscript.

Acknowledgements. The authors would like to thank GDR CNRS 3711 Cosm'Actifs and the Cosmetic Valley world cluster for their key role in promoting research in cosmetic sciences. We thank A. Edelman \& Associates for efficient English reviewing.

\section{Tables}

Due to technical limitations, Tables 1-4 are provided in the Supplementary Files section.

\section{References}

ANSM, Agence National de Sécurité du Médicament et des Produits de Santé (2012) Concentration de phénoxyéthanol dans les produits cosmétiques - Point d'information. https://ansm.sante.fr/Sinformer/Points-d-information-Points-d-information/Concentration-de-phenoxyethanol-dans-les-produitscosmetiques-Point-d-information

Bossis E, Lemanceau P, Latour X, Gardan L. The taxonomy of Pseudomonas fluorescens and Pseudomonas putida: current status and need for revision. Agronomie 20 (1):51-63

Briasco B, Capra P, Cozzi AC, Mannucci B, Perugini P (2016) Packaging evaluation approach to improve cosmetic product safety. Cosmetics 3(3): 32

Crozier A (2018a) Chapitre 14 : Efficacité protectrice microbiologique des packagings. In « Evaluation des Produits Cosmétiques : La Sécurité » M. Feuilloley N. Orange Eds. Cosmetic Valley. Chartres, France. ISBN : 978-2-490639-01-4. pp 255-264

Crozier A (2018b) Evaluation des performances des packs protecteurs. Point sur les travaux du GT EPMP de la Cosmetic Valley. 37ème Journée d'Etudes de I'ANEMCOLI. Lille, 10-11 octobre 2018

Devlieghere F, De Loy-Hendrickx A, Rademaker M, Pipelers P, Crozier A, De Baets B, Joly L, Keromen S (2015) A new protocol for evaluating the efficacy of some dispensing systems of a packaging in the microbial protection of water-based preservative-free cosmetic products. Int J Cosmet Sci 37(6):627-635

ECHA European Chemical Agency (2010) Member state committee draft support document for identification of boric acid as a substance of very high concern because of its CMR properties. https://echa.europa.eu/documents/10162/d51fd473-40ec-4831-bc2d-6f53bdf9cbbe 
European Community classification (2005) Risk group classification (prokaryotes). http://www.bacterio.net/-hazard.html

Feuilloley MGJ, Orange N (2018) Chapitre 15 : Interaction du cosmétique avec le microbiote cutané. In « Evaluation des Produits Cosmétiques : La Sécurité » M. Feuilloley N. Orange Eds. Cosmetic Valley, Chartres, France. ISBN : 978-2-490639-01-4. pp 266-276

Feuilloley M, Roullet F (2018) Towards a guideline for the evaluation of the microbiological protective activity of cosmetic packaging. Cosmetic360, Paris, 17-18 octobre 2018.

Halla N, Fernandes IP, Heleno SA, Costa P, Boucherit-Otmani Z, Boucherit K, Rodrigues AE, Ferreira ICFR, Barreiro MF (2018) Cosmetics preservation: A review on present strategies. Molecules 23(7): E1571

Herman A, Herman AP, Domagalska BW, Młynarczyk A (2013) Essential oils and herbal extracts as antimicrobial agents in cosmetic emulsion.Indian J Microbiol 53(2):232-237

ISO 11930 (2019) Cosmetics - Microbiology - Evaluation of the antimicrobial protection of a cosmetic product. https://www.iso.org/standard/75058.html

ISO 21149 (2006) Cosmetics - Microbiology - Enumeration and detection of aerobic mesophilic bacteria. https://www.iso.org/standard/35694.html

ISO 29621 (2017) Cosmetics-Microbiology-guidelines for the risk assessment and identification of microbiologically low-risk products. https://www.iso.org/standard/68310.html

Lundov MD, Moesby L, Zachariae C, Johansen JD (2009) Contamination versus preservation of cosmetics: a review on legislation, usage, infections, and contact allergy. Contact Dermatitis 60(2):70-78

Lum KT, Meers PD (1989) Boric acid converts urine into an effective bacteriostatic transport medium. J Infect 18(1):51-58

NF EN 29073-1 (1992) Textiles. Test methods for nonwovens. Part 1 : determination of mass per unit area. https://www.boutique.afnor.org/standard/nf-en-29073-1/textiles-test-methods-for-nonwovens-part1-determination-of-mass-per-unit-area/article/639660/fa024937

Papageorgiou S, Varvaresou A, Tsirivas E, Demetzos C (2010) New alternatives to cosmetics preservation. J Cosmet Sci 61(2):107-123

Regulation (EC) No 1223/2009 of the European Parliament and of the Council of 30 November 2009. Official Journal of the European Union L 342: 59-209. 22.12.2009. http://eurlex.europa.eu/ LexUriServ/LexUriServ.do? uri=0J:L:2009:342:0059:0209:en:PDF [1 November 2013]

Rowlands M, Blackwood L, Mas A, Cripps P, Crompton C, Burrow R (2011) The effect of boric acid on bacterial culture of canine and feline urine. J Small Anim Pract 52(10):510-514 
Sporer AJ, Kahl LJ, Price-Whelan A, Dietrich LEP (2017) Redox-based regulation of bacterial development and behavior. Annu Rev Biochem 86:777-797

Thompson RC, Moore CJ, Vom Saal FS, Swan SH (2009) Plastics, the environment and human health. Phil Trans R Soc B 364:2153-2166

Wilson, M (2005) The skin and its indigenous microbiota. In Microbial inhabitants of humans. Wilson M Ed. Cambridge University Press, Cambridge. pp 51-106

\section{Figures}

A
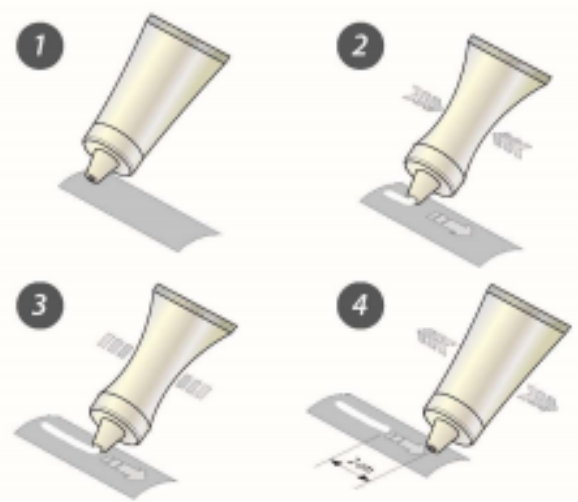

B

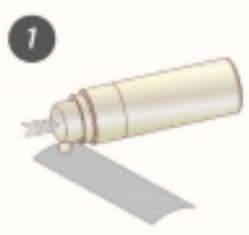

2

3
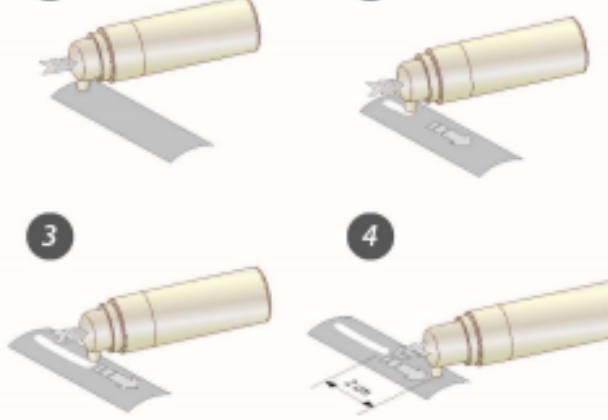

4

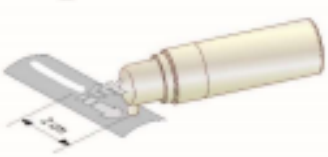

\section{Figure 1}

Illustration of the contamination procedure developed to reproduce natural contamination due to skin contact under standardized conditions. For flexible packaging (A), the nozzle of the pack is pressed in contact with the contaminated compress $(106 \mathrm{CFU} / \mathrm{mL})(1)$ and the dose (as defined by the provider) is released (2). When it is completely restituted (3), the pressure on the packaging is relieved and the movement is prolonged for a distance of $2 \mathrm{~cm}$ (4) to mimic manual wiping of the tip. This procedure can be adapted to rigid packaging $(B)$ by replacing the pressure on the side of the pack by actuation of the delivery pump. 

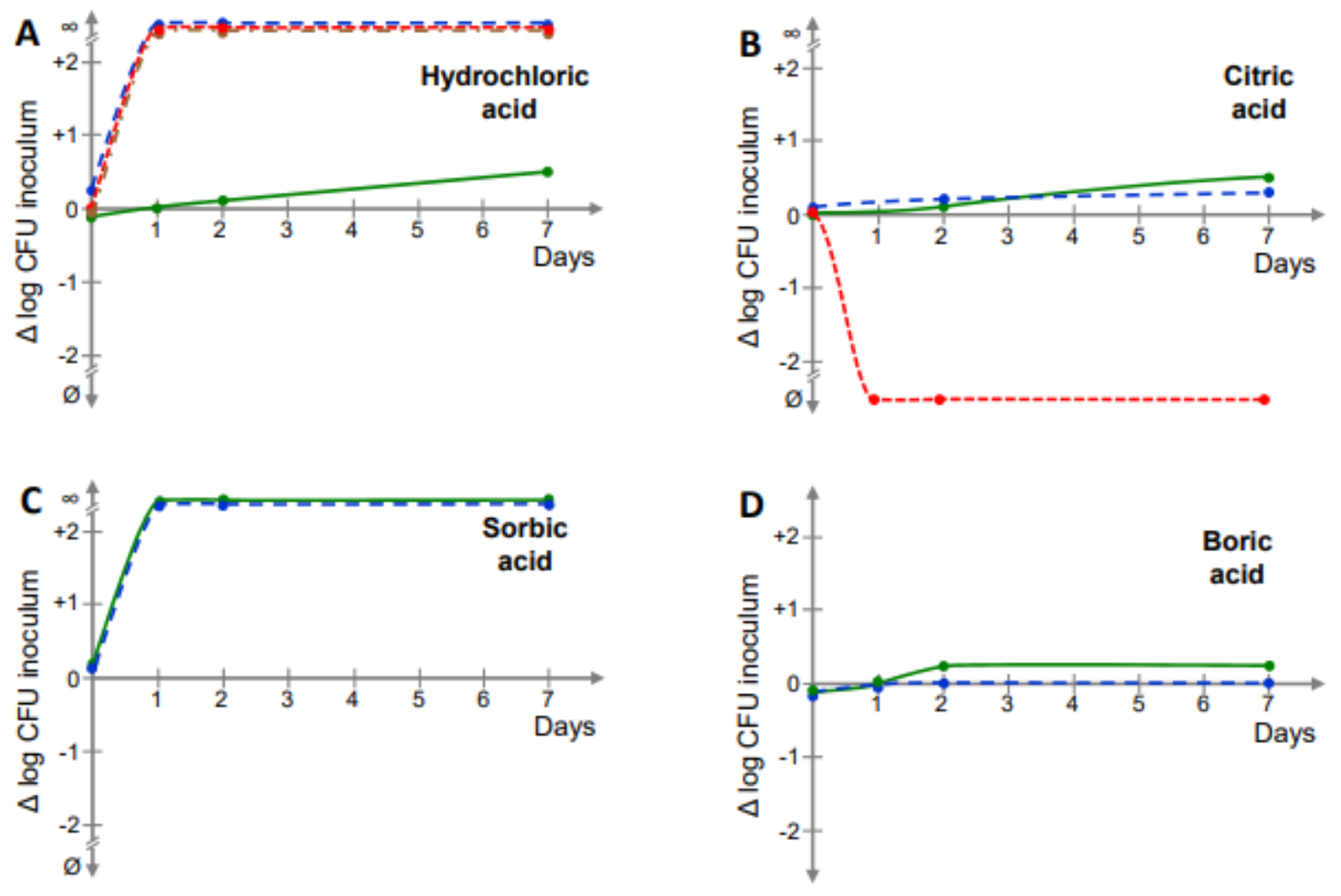

\section{Figure 2}

Comparison of the bacteriostatic activities of media produced in the presence of different acids. The evolution of the contamination was measured over 7 days and was expressed as the logarithmic variation of the initial inoculum ( $\triangle \log \mathrm{CFU}$ : colony-forming unit). A. Hydrochloric acid $\mathrm{pH}=5.39$, $\mathrm{pH}=5.45, \mathrm{pH}=5.63$, and $\mathrm{pH}=5.91$. B. Citric acid $\mathrm{pH}=4.79, \mathrm{pH}=4.81$, and $\mathrm{pH}=4.94$.C. Sorbic acid $0.05 \%$ and $0.1 \%$. D. Boric acid $0.84 \%(\mathrm{pH}=5.41)$ and $0.26 \%(\mathrm{pH}=6.00)$. All experiments were performed in triplicate and completed with tests at higher and lower concentrations (not shown). 

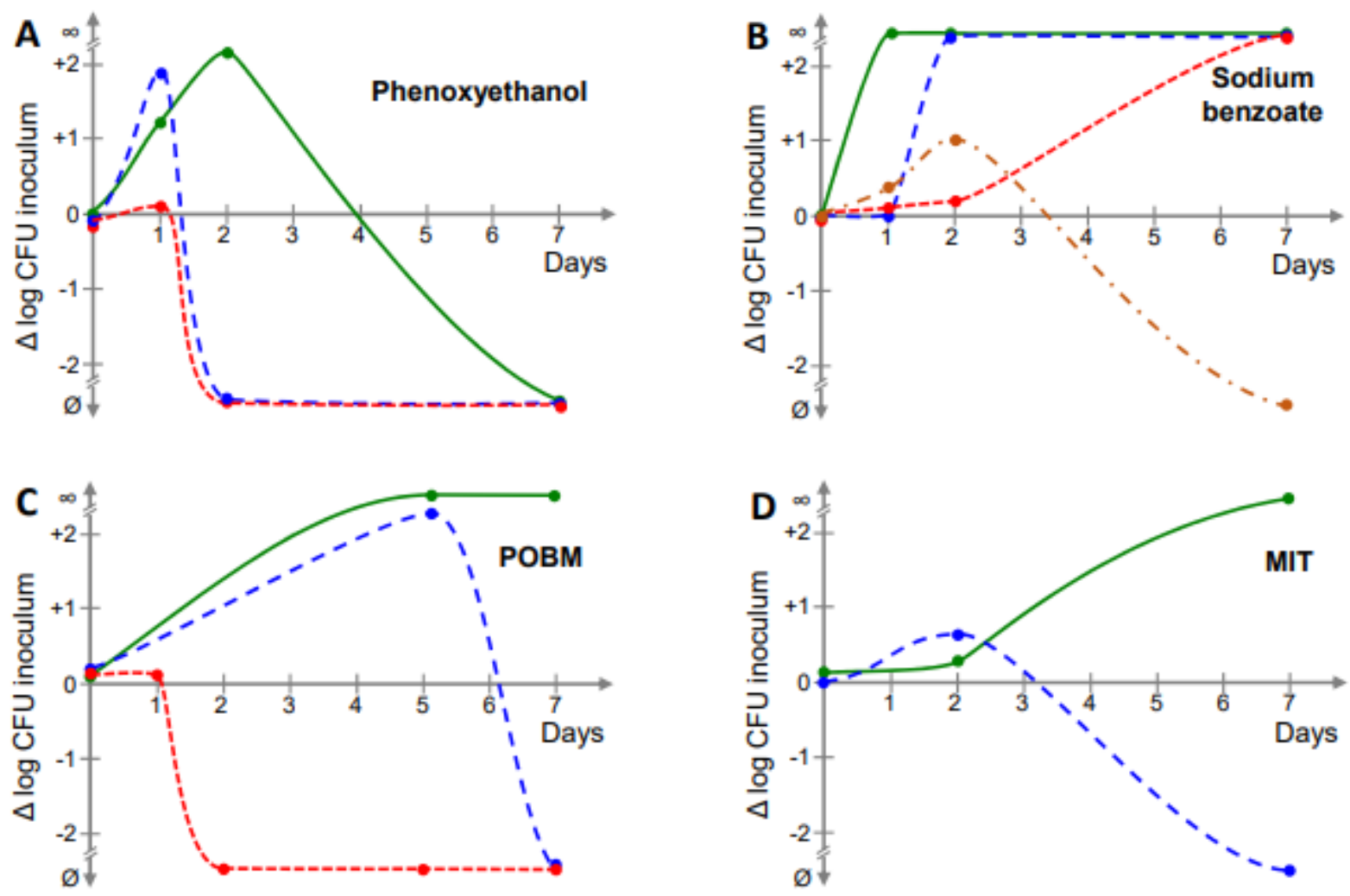

\section{Figure 3}

Comparison of the bacteriostatic activities of media produced in the presence of common organic preservatives. The evolution of the contamination was measured over 7 days and was expressed as the logarithmic variation of the initial inoculum ( $\Delta \log C F U$ : colony-forming unit). A. Phenoxy ethanol $0.3 \%$, $0.32 \%$ and $0.35 \%$. B. Sodium benzoate $0.01 \%, 0.03 \%, 0.04 \%$, and $0.05 \%$. C. POBM (methyl paraben) $0.1 \%, 0.12 \%$ and $0.15 \%$. D. MIT (methyl isothizolinone) $0.001 \%$ and $0.002 \%$. All experiments were performed in triplicate and completed with tests at higher and lower concentrations (not shown). 


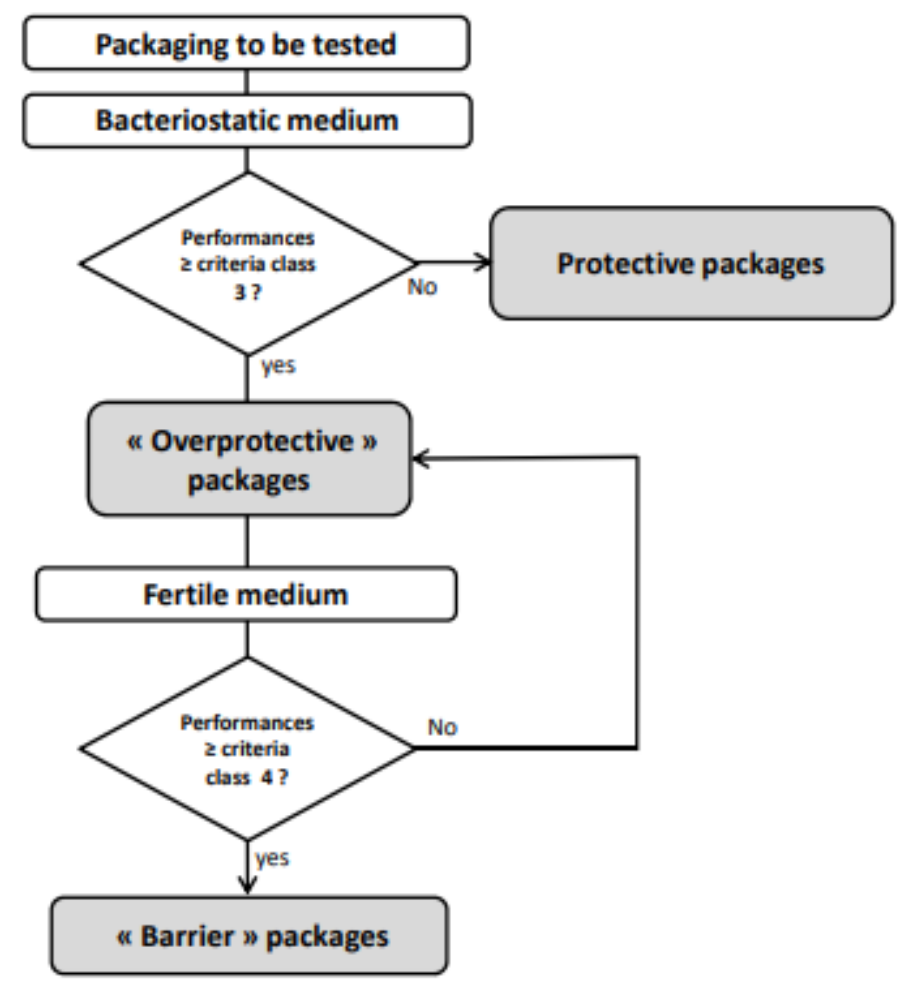

Figure 4

Flowchart established to classify the microbial protection potential of packaging.

\section{Supplementary Files}

This is a list of supplementary files associated with this preprint. Click to download.

- Table4.pdf

- Table1.pdf

- Table2.pdf

- Table3.pdf 